\title{
Neurovascular Oxidative Stress and Autonomic Modulation Contributing to QT Interval Variations in Acute Large Artery Ischemic Stroke
}

\author{
Suwanprasert $\mathrm{K}^{1 *}$, Chanbenjapipu $\mathrm{P}^{2}$ and \\ Muengtaweepongsa $\mathbf{S}^{3}$ \\ ${ }^{1}$ Department of Preclinical Sciences, Thammasat \\ University (Rangsit Campus), Thailand \\ ${ }^{2}$ Medical Engineering Program, Thammasat University \\ (Rangsit Campus), Thailand \\ ${ }^{3}$ Department of Medicine, Thammasat Univrersity \\ (Rangsit Campus), Thailand \\ *Corresponding author: Suwanprasert K, Department \\ of Preclinical Sciences, Thammasat University (Rangsit \\ Campus), Thailand
}

Received: July 14, 2017; Accepted: August 08, 2017; Published: October 13, 2017

\begin{abstract}
Background: Acute stroke can disturb autonomic regulation and promoting oxidative stress. Prolongation of QT interval is very common in stroke and related with stroke recurrent and sudden death. In this study, we hypothesize that neurovascular oxidative stress and autonomic modulation contribute to QT interval variation.
\end{abstract}

Method: Sixty five acute large artery ischemic strokes were matched control and recruited. Three patient groups of QT interval variation were classified as short QTc (SQTc, age 61.31 $\pm 1.96, \mathrm{n} 36$ ), normal QTc (NQTc, age 63.60 \pm 2.83 , n 15) and long QTc (LQTc, age $62.90 \pm 4.19$, n 14). Plasma nitric oxide (NO) and hydrogen peroxide $\left(\mathrm{H}_{2} \mathrm{O}_{2}\right)$ were measured by electrochemistry technique. Heart rate variability (HRV) was assessed by Power Lab and Kubios Program. Pulse transit time (PTT) was recorded by Lead II and finger pulse wave. Stenotic flow velocity and intima/media thickness (IMT) ratio were measured by Doppler ultrasound.

Results: Profound lower $\mathrm{NO}$ is found in all groups. Highest level of $\mathrm{H}_{2} \mathrm{O}_{2}$ is evident in LQTc and differs significantly from those in SQTC and NQTC. Greater $\mathrm{HR}$, blood pressure, pulse pressure and reduced pulse transit time were found in LQTC. Insignificant difference of stenotic flow velocity and IMT ratio was demonstrated in all QTc groups. In LQTc, greater LF/HF ratio and SD2/SD1 suggest predominant sympathetic drive over parasympathetic activity. Greater scatter gram of Poincare' plot corresponding with low value of sample entropy in LQTc indicate well organized complexity of autonomic modulation.

Conclusion: Neurovascular oxidative stress and autonomic modulation contribute to QT interval variation during acute stroke.

Keywords: QT interval; Autonomic modulation; Neurovascular oxidative stress; Acute ischemic large artery stroke

\section{Abbreviation}

LDL: Low Density Lipoprotein; HDL: High Density Lipoprotein; HT: Hypertension, DM: Diabetes Mellitus; NSSF: Non Significant Stenosis Flow; SSF: Significant Stenosis Flow; MIT: Media Intima Thickness; ACEI: Angiotensin II-Converting Enzyme Inhibitor; SDNN(ms): Standard Deviation of all NN Intervals; PNN50(\%): Number of adjacent NN intervals that differ by $50 \mathrm{~ms} /$ total number of NN intervals; LF(nu): Low Frequency Range (0.04-0.15Hz); HF(nu): High Frequency Range $(0.15-0.4 \mathrm{~Hz})$; SD1(ms): Standard Deviation of Points Perpendicular to the Line of Identity; SD2(ms): Standard Deviation of Points along to the Line of Identity; SampEn: Sample Entropy

\section{Introduction}

Acute stroke can disturb brain-heart axis shift through central autonomic control and promoting advancing oxidative stress from ischemia resulting in electrocardiographic abnormalities, cardiac arrhythmias, myocardial injury and ultimately sudden death [15]. Previous studies have reported that atrial arrhythmia (AF), prolonged QT interval and tachycardia are the most common cardiac arrhythmia during ischemic stroke with the severity of recurrent stroke and sudden death [4-6]. Prolonged QTc interval has been reported in $23 \%-45 \%$ of patients during acute stroke [7]. Cardiacautonomic modulation by increasing sympathetic activity and/or decreasing parasympathetic activity is commonly found and shows significant characteristic of reduced heart rate variability (HRV) $[8,9]$. Autonomic nervous system (ANS) itself is not linear function and it has been argued that it is nonlinear function feature since the concept of autonomic control is a closed loop model with the sympathetic and parasympathetic limbs acting in a reciprocal fashion in order to maintain homeostasis [10]. Nonlinear function of HRV is the most powerful tool exploring a shift of brain-heart axis and ultimately modulation of ANS [11,12]. Recently, meaningful missing beats of $\mathrm{R}-\mathrm{R}$ intervals have been shown contributing abnormal QTc and AF in large artery ischemic stroke [13]. Moreover, an inverse correlation between SDNN, parasympathetic HRV parameters (rMSSD and pNN50) and atrial fibril atory rate (AFR) of atrial activity has been reported in stroke-AF patients, and this is a possible link between AFR and autonomic modulation [14]. The QT interval prolongation
Austin J Cerebrovasc Dis \& Stroke - Volume 4 Issue 5 - 2017 ISSN : 2381-9103 | www.austinpublishing group.com Suwanprasert et al. @ All rights are reserved
Citation: Suwanprasert K, Chanbenjapipu P and Muengtaweepongsa S. Neurovascular Oxidative Stress and Autonomic Modulation Contributing to OT Interval Variations in Acute Large Artery Ischemic Stroke. Austin J Cerebrovasc Dis \& Stroke. 2017; 4(5): 1071. 
is considered a marker of electrical instability of the ventricular myocardium and may be associated with an increased incidence of rhythm disturbances, ventricular arrhythmias and sudden death [15]. Ventricular repolarization in QT interval might affect the next $\mathrm{P}$ wave on the second ECG cycle which it means there is a possible abnormal $\mathrm{P}$ wave throughout EKG tracing and behaves the missing beat in R-R peak. R-R intervals (RRIs) are a series of beat to beat of heart contraction which composes of two cardiac cycles. Hence, abnormal ECG waves behave as any noise or showing missing beats may influence cardiac autonomic modulation. During acute phase of stroke, an apoptotic and neuro inflammatory response are developed as a result of the stimulatory influences of ischemia such as reactive oxygen species (ROS) which presenting with further abundant NO production from inducible nitric oxide synthase (iNOS) activity from astrocytes and macrophages [16]. Normally, most NO in brain derives from endothelial NOS (eNOS) and neuronal NOS (nNOS) in neurovascular unit during neurovascular coupling process [17]. Recently, profound lower circulating NO has reported during acute ischemic stroke [18]. Moreover, neurovascular coupling assessed by Heart Rate Variability (HRV), circulating $\mathrm{NO}$ and $\mathrm{H}_{2} \mathrm{O}_{2}$ during cerebrovascular reactivity in normal person has reported suggesting the interaction of signaling molecules of redox signal through $\mathrm{NO}$ and $\mathrm{H}_{2} \mathrm{O}_{2}$ cerebral blood flow and autoregulation [19]. $\mathrm{H}_{2} \mathrm{O}_{2}$ is stable reactive oxygen species (ROS) and causes relaxations of large cerebral arteries in part by activation of arachidonic acid metabolism via cyclooxygenase pathway with subsequent increase in cAMP levels and activation of potassium channels [20]. It has been reported that microglia proliferation following brain damage is regulated by $\mathrm{H}_{2} \mathrm{O}_{2}$ from NADPH oxidase [21]. Recently, ROS may raise BP via activation of the sympathetic nervous system and mediated in part by downregulation of nNOS and NO synthesis [22]. There are several possible ways for ROS to induce arrhythmia such focal activity and reentry, ionic currents, promoting cardiac fibrosis and impaired gap junction function which resulting in reduced myocyte coupling and facilitation of reentry [23]. In this study, we hypothesize that neurovascular oxidative stress by $\mathrm{NO}$ and $\mathrm{H}_{2} \mathrm{O}_{2}$ and cardiac autonomic modulation contribute to QT interval variations during acute large artery ischemic stroke.

\section{Methods}

\section{Subjects}

Sixty five acute large artery stroke patients (38 male, 27 female, age 62.09 \pm 3.13 ) were recruited and classified as normal QTc (NQTc), short QTc (SQTc) and long QTc (LQTc) of large artery ischemic stroke patients (by fMRI studies) with matching control in general characteristics and carotid stenosis flow velocity whom were recruited from 165 acute ischemic stroke. Thirty six short QTc, fifteen normal QTc and eleven long QTc (mean age 61.31 \pm 1.96 , $63.60 \pm 2.83$ and $62.90 \pm 4.19$ years respectively) were first ever stroke and admitted within $24 \mathrm{~h}$ (mean $7.1 \pm 2.2 \mathrm{~h}$ ) of the onset of symptoms. All participants gave their written informed consent to participate in the study which approved by the local Ethical Committee (MTU-ECIM-018154)

\section{Measurement of $\mathrm{NO}$ and $\mathrm{H}_{2} \mathrm{O}_{2}$}

Plasma samples were withdrawn and centrifuged from the whole blood, collected and then measured for $\mathrm{NO}$ and $\mathrm{H}_{2} \mathrm{O}_{2}$ by electrochemical technique at the day of admission [24,25]. NO and $\mathrm{H}_{2} \mathrm{O}_{2}$ concentrations were measured by using amino-700 probe and $\mathrm{HP}-250 \mathrm{H}_{2} \mathrm{O}_{2}$ electrochemical sensor, respectively (inNO nitric oxide measuring system, model inNO-T S/N 3782-G, Tampa, Fl, USA).

\section{Assessment of common carotid artery (CCA) intima- media thickness (IMT) by carotid duplex scan}

Common carotid artery-intima media thickness (CCA-IMT) was assessed by a high-resolution ultrasound B-mode on Philips iE33 with a $7.5 \mathrm{MHz}$ linear probe. The carotid arteries were examined for atherosclerotic plaques, which defined as focal widen that relative to adjacent segments or with projection into vascular lumen of calcified lesion or a combination of calcification and non-calcified material [26]. For studying carotid wall thickness, ultrasonographic examination was conducted with subjects lying in supine position with the head turned $45^{\circ}$ to the left or right. Longitudinal images of the left and right common carotid arteries (CCA) were acquired. The near and far walls of the carotid arteries showed as two bright white lines separated by a hypo echogenic space. One frozen images of far wall IMT were acquired from both of right and left CCA. IMT was the distance between the leading edge of the upper intima interface to the leading edge of the upper adventitia interface as intima media. Measurement of CCA-IMT was done offline within $1 \mathrm{~cm}$ which it was proximal to the carotid bulb.

\section{QTc measurement}

The QT interval was measured at a speed of $25 \mathrm{~mm} / \mathrm{s}$, the EKGs were accepted for QT evaluation if the heart rate was between 60 and 100 beats $/ \mathrm{min}$. The QT interval was corrected for heart rate by Bazett's formula $(\mathrm{QTc}=\mathrm{QT} / \sqrt{R R})$ [27]. A short QTc, normal QTc and prolonged QTc were defined as from lesser than360, 360-390 and greater than 400 milliseconds, respectively [28].

\section{Heart rate variability (HRV)}

The patients remained at rest for $10 \mathrm{~min}$ in the supine position and were instructed to breathe spontaneously. Then, the instantaneous R-R intervals (RRI) of lead II EKG were recorded continuously for 15 min using Power Lab [29].

Data acquisition: The EKG recordings were acquired with a sampling rate of $1000 \mathrm{~Hz}$ using lead II for 15-minute long duration within 24 hours after admission [29]. All recordings were made in similar conditions with subjects maintained at rest and in a comfortable position. The EKG interpretation with diagnostic confirmation was done by 2 independent physicians. The EKG recording files were further tested in a blinded fashion. RRIs detection was processed by using Lab Chart Pro software. Base on filtering process order in software (eg. band-pass, median, derivative, smoothing and normalize process), the marker were located at peak of $\mathrm{R}$ wave then they were manually rechecked. The $\mathrm{R}$ to $\mathrm{R}$ interval was extracted to format file for HRV analysis.

Analysis of heart rate variability: The data sets that preprocessing from the Lab Chart Pro software were transferred as input data to Heart Rate Variability Analysis Software (Kubios-HRV version 2.0, University of Kuopio, Finland) and then analyzed for timedomain HRV parameters, frequency domain and non- linear HRV parameters [30]. 
Table 1: Characteristics of SQTc, NQTC and LQTC.

\begin{tabular}{|c|c|c|c|c|}
\hline Characteristics & $\begin{array}{c}\text { SQTc } \\
(n=36) \\
\text { mean } \pm \text { SEM }\end{array}$ & $\begin{array}{c}\text { NQTc } \\
(n=15) \\
\text { mean } \pm \text { SEM }\end{array}$ & $\begin{array}{c}\text { LQTc } \\
(n=14) \\
\text { mean } \pm \text { SEM }\end{array}$ & ${ }^{*} p<0.05$ \\
\hline Age & $61.31 \pm 1.96$ & $63.60 \pm 2.83$ & $62.90 \pm 4.19$ & NS \\
\hline $\begin{array}{c}\text { Sex } \\
\text { Male/ Female }\end{array}$ & 24,12 & 8,7 & 6,8 & NS \\
\hline LDL (mg/dl) & $137.82 \pm 10.50$ & $99.41 \pm 11.85$ & $102.42 \pm 17.19$ & NS \\
\hline $\mathrm{HDL}(\mathrm{mg} / \mathrm{dl})$ & $48.34 \pm 3.45$ & $56.63 \pm 5.99$ & $49.14 \pm 5.27$ & NS \\
\hline $\mathrm{HT}, \mathrm{n}(\%)$ & $16(44.44 \%)$ & $6(40.00 \%)$ & $4(28.57 \%)$ & NS \\
\hline DM, n (\%) & $1(2.77 \%)$ & $1(6.67 \%)$ & $2(14.29 \%)$ & NS \\
\hline DM, HT, n (\%) & $5(13.89 \%)$ & $2(13.33 \%)$ & $3(21.43 \%)$ & NS \\
\hline \multicolumn{5}{|l|}{ Carotid stenosis, n (\%) } \\
\hline NSSF, n (\%) & $24(66.67 \%)$ & $9(60.00 \%)$ & $10(71.42 \%)$ & NS \\
\hline SSF, n (\%) & $12(33.33 \%)$ & $6(40.00 \%)$ & $4(28.58 \%)$ & NS \\
\hline MIT ratio: Right side & $0.71 \pm 0.03$ & $0.65 \pm 0.05$ & $0.73 \pm 0.07$ & NS \\
\hline MIT ratio: Left side & $0.66 \pm 0.03$ & $0.68 \pm 0.04$ & $0.73 \pm 0.06$ & NS \\
\hline \multicolumn{5}{|l|}{ History medication } \\
\hline ACEI ,n & $1(2.78 \%)$ & 0 & 0 & NS \\
\hline Bblocker ,n & $1(2.78 \%)$ & 0 & 0 & NS \\
\hline $\mathrm{Ca}^{2+}$ blocker, $\mathrm{n}$ & $1(2.78 \%)$ & 0 & 0 & NS \\
\hline
\end{tabular}

NS; Non-Significant Difference

\section{Hemodynamic study}

Blood pressure (BP) and heart rate (HR): Blood pressure and heart rate were measured by non- invasive automate blood pressure monitor (IntelliVue MP40, Philips, Netherland) in stroke unit. Then, blood pressure is calculated to pulse pressure and mean arterial blood pressure.

Pulse transit time (PTT): PTT is a time of pulse pressure wave from left ventricle contraction to peripheral vessel. These can be determined by measuring times during $\mathrm{R}$ wave peak of QRS complex to pulse wave peak of the periphery finger tips [31]. In this study, lead II EKG and finger pulse wave were simultaneously recorded by the Power Lab systems (AD Instruments) with a sampling rate 1,000/s consecutive 2-5 minutes and then analyzed 10 windows by Lab chart program (AD Instruments).

\section{Statistical analysis}

The variables were expressed as mean \pm standard error of mean (SEM). Data were analyzed by one way ANOVA. A value of $\mathrm{P}<0.05$ was considered statistically significant difference.

\section{Results}

\section{Classification of QTc variation: SQTC, NQTc and LQTC}

By using Bazett's method, QT intervals of sixty five stroke patients were classified as short QTc (SQTc, $<360 \mathrm{msec}$ ), normal QTc (NQTc, $360-390 \mathrm{msec}$ ) and long QTc or prolonged QTc (LQTc $\geq 400$ $\mathrm{msec}$ ) [28]. The patients were matched control in all aspect of general characteristics including coronary stenosis and flow velocity which they showed insignificant difference Table 1.

\section{Neurovascular oxidative stress, cardio-hemodynamic and HRV analysis}

Different levels of circulating $\mathrm{NO}$ and $\mathrm{H}_{2} \mathrm{O}_{2}$ concentrations
Table 2: Results of neurovascular oxidative stress through $\mathrm{NO}$ and $\mathrm{H}_{2} \mathrm{O}_{2}$, cardiohemodynamic and HRV analysis in S QTC, N QTC and L QTC.

\begin{tabular}{|c|c|c|c|}
\hline Variables & $\begin{array}{c}\text { SQTc } \\
(n=36) \\
\text { mean } \pm \text { SEM }\end{array}$ & $\begin{array}{c}\text { NQTc } \\
(n=15) \\
\text { mean } \pm \text { SEM }\end{array}$ & $\begin{array}{c}\text { LQTc } \\
(n=14) \\
\text { mean } \pm \text { SEM }\end{array}$ \\
\hline $\mathrm{SBP}(\mathrm{mmHg})$ & $151.52 \pm 3.68$ & $139.10 \pm 3.76$ & $161.44 \pm 6.12^{\#}$ \\
\hline $\mathrm{DBP}(\mathrm{mmHg})$ & $84.24 \pm 2.18$ & $76.69 \pm 3.28$ & $89.70 \pm 4.86^{\#}$ \\
\hline $\mathrm{HR}$ (rate/min) & $69.60 \pm 2.05^{\circ}$ & $69.2667 \pm 2.08$ & $82.25 \pm 5.41^{\#}$ \\
\hline $\mathrm{PP}(\mathrm{mmHg})$ & $66.60 \pm 2.87 \cdot$ & $67.93 \pm 3.74$ & $111.80 \pm 4.85^{\# \#}$ \\
\hline $\operatorname{MABP}(\mathrm{mmHg})$ & $107.30 \pm 2.45$ & $103.44 \pm 3.90$ & $109.30 \pm 5.05$ \\
\hline PTT (sec) & $0.24 \pm 0.00 *$ & $0.24 \pm 0.00$ & $0.20 \pm 0.00^{\#}$ \\
\hline $\mathrm{NO}(\mathrm{nM} / \mathrm{L})$ & $27.36 \pm 1.22$ & $24.29 \pm 1.45$ & $23.33 \pm 2.86$ \\
\hline $\mathrm{H}_{2} \mathrm{O}_{2}(\mathrm{uM} / \mathrm{L})$ & $32.17 \pm 2.64$ & $31.76 \pm 2.66$ & $40.10 \pm 5.88^{\#}$ \\
\hline $\begin{array}{l}\text { HRV analysis } \\
\text { SDNN(ms) }\end{array}$ & $23.45 \pm 2.17$ & $29.90 \pm 4.81$ & $19.97 \pm 2.17$ \\
\hline pNN 50(\%) & $15.04 \pm 3.72$ & $22.84 \pm 8.51$ & $33.25 \pm 14.89^{\circ}$ \\
\hline LF(n.u.) & $46.25 \pm 2.58$ & $55.73 \pm 3.33$ & $72.30 \pm 5.36^{\# \#}$ \\
\hline HF(n.u.) & $58.25 \pm 2.73$ • & $52.23 \pm 4.28$ & $27.69 \pm 5.35^{\# \#}$ \\
\hline LF/HF ratio & $0.87 \pm 0.08 \cdot$ & $1.42 \pm 0.23$ & $4.17 \pm 0.94^{\# \#}$ \\
\hline $\mathrm{SD} 1(\mathrm{~ms})$ & $16.79 \pm 1.41$ & $19.72 \pm 3.76$ & $11.19 \pm 1.03^{\#}$ \\
\hline $\mathrm{SD} 2(\mathrm{~ms})$ & $31.26 \pm 6.36$ * & $46.58 \pm 6.57$ & $48.47 \pm 6.79 *$ \\
\hline SD2/SD1 & $2.44 \pm 0.20^{\circ}$ & $2.19 \pm 0.31$ & $3.74 \pm 0.56^{\#}$ \\
\hline$\alpha 1$ & $0.90 \pm 0.04$ & $0.93 \pm 0.06$ & $0.87 \pm 0.08$ \\
\hline$\alpha 2$ & $0.97 \pm 0.05$ & $0.94 \pm 0.07$ & $0.87 \pm 0.08$ \\
\hline$\alpha 1 / \alpha 2$ & $0.96 \pm 0.05$ & $1.02 \pm 0.05$ & $1.18 \pm 0.17$ \\
\hline SampEn & $1.44 \pm 0.04 \cdot$ & $1.43 \pm 0.12$ & $0.72 \pm 0.19^{\# \#}$ \\
\hline
\end{tabular}

* $p<0.05$ and ** $p<0.01$

"Significant Difference (S QTcvs N QTc)

\#Significant Difference (N QTcvs L QTc)

-Significant Difference (S QTcvs L QTC)

a1 Scaling Exponent $\alpha 1(n<11)$ of Detruded Fluctuation Analysis $\alpha 2$ Scaling Exponent $\alpha 2(\mathrm{n}<11)$ of Detruded Fluctuation Analysis

during $24 \mathrm{~h}$ acute stroke in three QTc variations are evident in Table 2 and Figure1. HRV analysis is demonstrated in Table 2.

During acute stroke NO gradually reduced from S QTcto LQTc as shown in Figure 2. Meanwhile, gradual increase in $\mathrm{H}_{2} \mathrm{O}_{2}$ was evident and showed significant difference between S QTc and L QTc (31.76 \pm 2.66 vs. $40.10 \pm 5.88)$. The findings suggest an overwhelmed ROS during ischemia and indicate the state of neurovascular oxidative stress [19]. Characterized feature of advancing oxidative stress is reducing $\mathrm{NO}$ caused by endothelial dysfunction, inactivation of NOS enzymes and NO depletion [18]. Additionally, increasing $\mathrm{H}_{2} \mathrm{O}_{2}$ confirms inactivation of SOD caused by an overwhelmed free radicals and $\mathrm{H}_{2} \mathrm{O}_{2}$ derived-glia activation, another source of synthesis, induced by neuronal damage.

As shown in Figure 2, cardio-hemodynamic response during acute stroke was evident and showed the significant differences among SQTc, NQTc and LQTc groups. Higher HR was significantly differed in L QTc (L QTc vs. N QTc; L QTc vs. S QTc). This result indicates the influence of ANS upon heart rate. Corresponding with an increasing HR, greater pulse pressure Figure 2 from high systolic blood pressure and diastolic blood pressure and lower pulse transit time (PTT) Table 2 in LQTc may be from overactivity of sympathetic drive resulting activation of catecholamine release. 

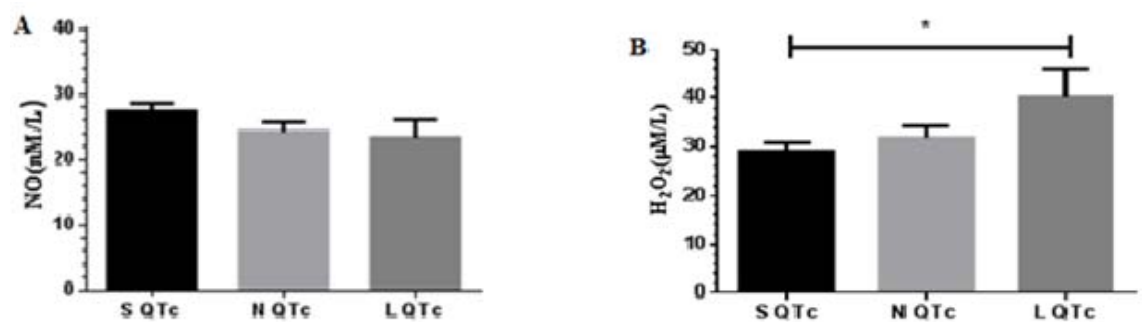

Figure 1: Comparison of $\mathrm{NO}(\mathrm{A})$ and $\mathrm{H}_{2} \mathrm{O}_{2}$ (B) in SQTc, NQTc and LQTc during acute stroke. * $\mathrm{p}<0.05$
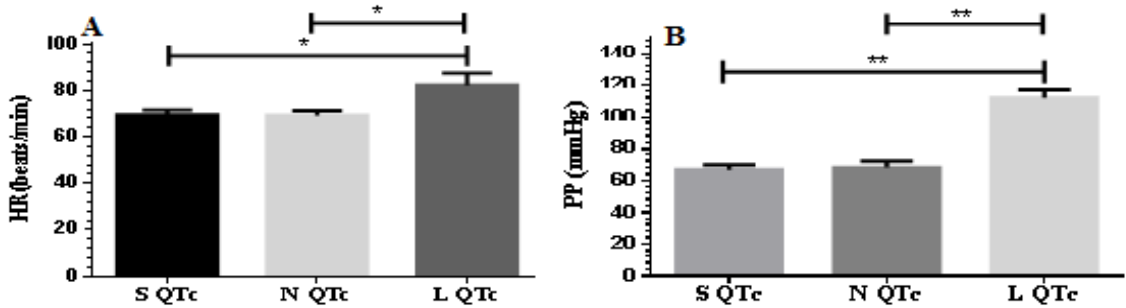

Figure 2: $(A)$ Greater values of heart rate $(H R)$ and $(B)$ Pulse pressure $(P P)$ in LQTc compared with SQTc and NQTc. " $p<0.05$; "* $p<0.01$

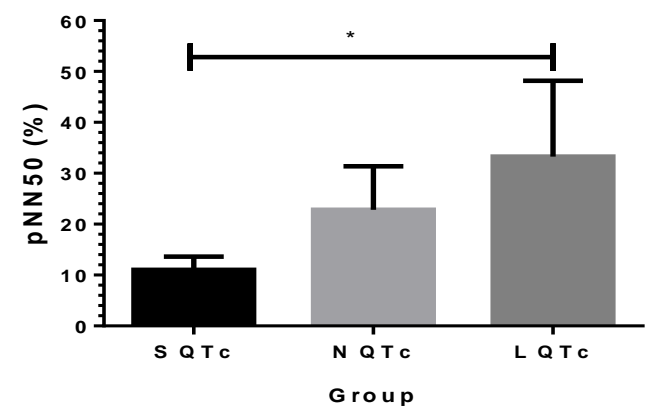

Figure 3: pNN 50(\%) of S QTc, N QTC and L QTc during acute stroke."p<0.05.

pNN 50(\%) of L QTc was significant high value compared with S QTc and N QTc (Figure 3). This finding indicates high vagal outflow in order to increase the quantitation and qualitative of sympathovagal balance. This result strongly confirms the greater value of LF/HF ratio in Figure 4 and sample entropy in Figure 7. Power spectral density (PSD) analysis provides the basic information of how power (variance) distributes as a function of frequency domain: very low frequency or VLF $(\leq 0.04 \mathrm{~Hz})$, low frequency or LF $(0.04-0.15 \mathrm{~Hz})$, and high frequency or HF (0.15-0.4 Hz) components. As shown in Figure 4, LF/HF ratio was significantly elevated compared between S QTc and N QTc $(0.87 \pm 0.08 v s 1.42 \pm 0.23)$ and L QTc (4.17 \pm 0.94$)$ with S QTc and N QTc. Corresponding HF and LF showed a significant difference among groups as well. These results indicate both quantitation and qualitative autonomic modulation in term of dynamic changes of parasympathetic and sympathetic drives.

\section{Nonlinear HRV analysis: Poincare plot analysis}

Since HRV is controlled by complex regulation mechanisms, it undoubtedly contains nonlinear properties. RR interval Poincare plot analysis is one of newer nonlinear methods which represent a quantitative visual technique (Figure 5,6). The Poincare plot is a scatter plot of the current RR interval plotted against the preceding $\mathrm{RR}$ interval. Points below the line at $45^{\circ}$ to the normal axis or the so
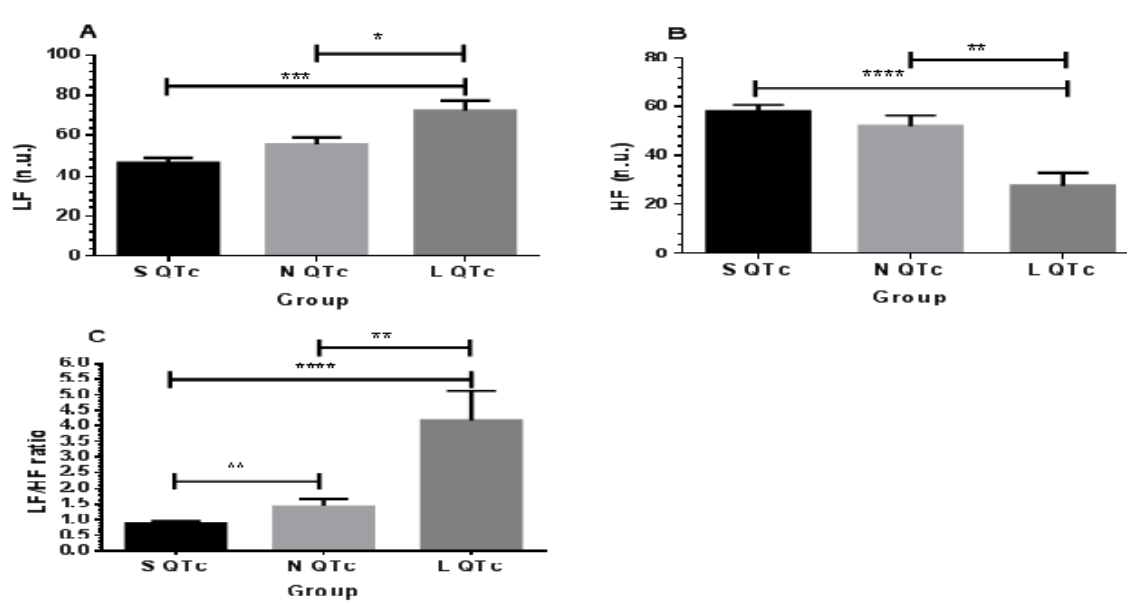

Figure 4: Frequency domain of HRV: LF (A), HF (B) and LF/HF ratio (C) in SQTc, NQTc and LQTc. " $p<0.05 ;{ }^{* *} p<0.01 ;{ }^{* * *} p<0.001$ 

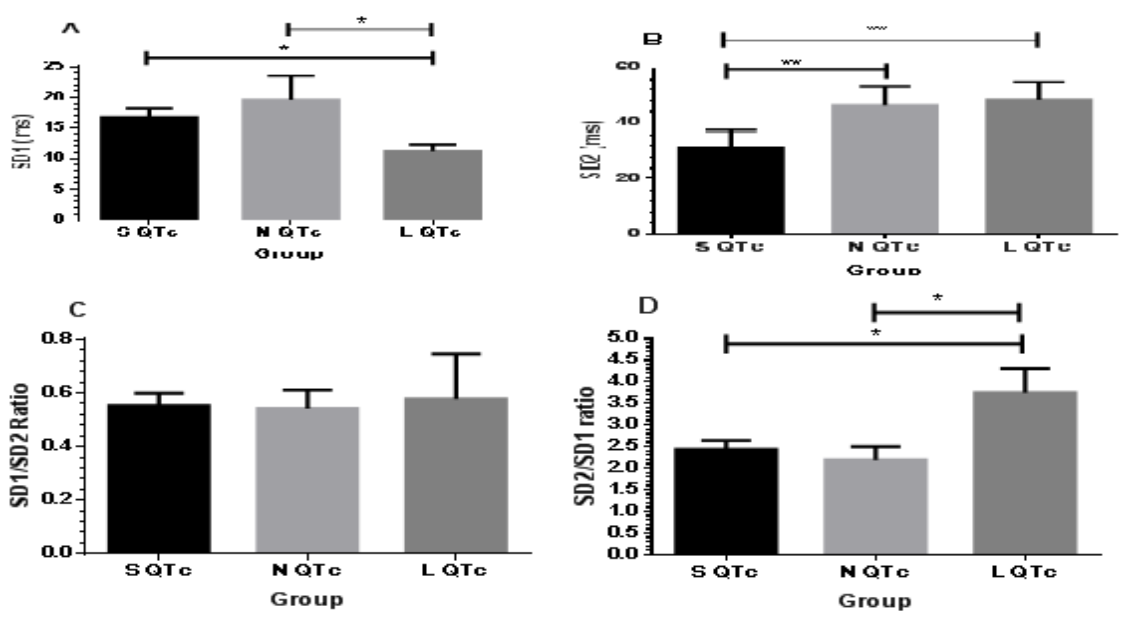

Figure 5: Comparison of SD1 (A), SD2 (B), SD1/SD2 ratio (C) and SD2/SD1 ratio (D) in SQTc, NQTc and LQTc.
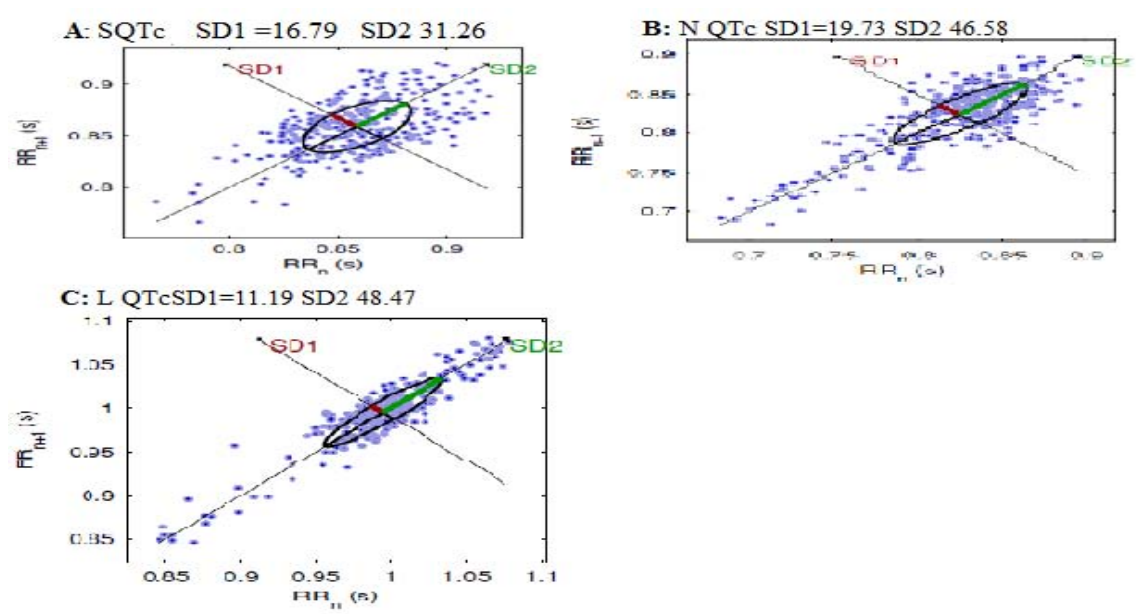

Figure 6: Poincare's Plot from representative groups of (A) SQTc, (B) N QTc and (C) L QTc during acute stroke.

called line of identity indicate RR intervals that are shorter than the preceding RR interval, and points above the line of identity indicate longer RR intervals than the previous. The dispersion of points along the perpendicular axis to the line of identity reflects short-term variability, quantified by the standard deviation of the distances the points lie from the line of identity (SD1). SD1 relates to the standard deviation of the successive differences of the RR intervals (SDSD) or MSSD which is mainly caused by RSA and mediated by the parasympathetic system or vagal activity. The standard deviation of points along the line of identity (SD2) reflects the standard deviation of the RR intervals, and has shown to be related with long-term variability [32-34].

Distribution of SD1, SD2 and SD2/SD1 was demonstrated in Figure 5. Profound suppression of SD1 was noticed in L QTc and it differed significantly compared with S QTc and N QTc. Moreover, $\mathrm{SD}_{2}$ in L QTc was higher than S QTc and NQTc and showed significant difference with SQTc. SD2 trend was similar to SD2/SD1 ratio. Greater value of SD2/SD1 ratio was found in L QTc and differed significantly with S QTc and N QTc. The findings indicate remarkable suppression of parasympathetic drive resulting predominant sympathetic activity

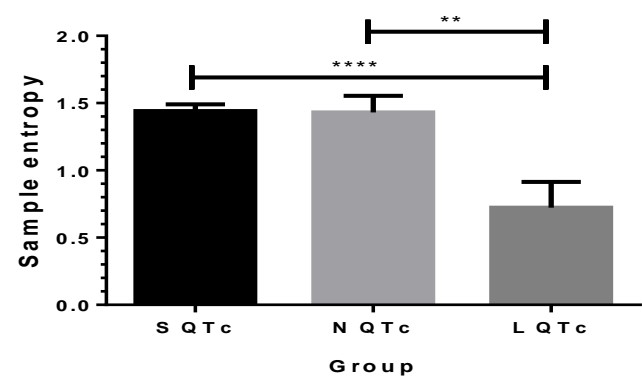

Figure 7: Sample entropy in SQTc, NQTc and LQTc strokes. " $p<0.01$; ${ }_{* * * *} p<0.0001$

in L QTc. In vice versa, less suppression of parasympathetic in SQTc and NQTc causes less quantitation of sympathetic activity (by SD2/ SD1) in N QTc and S QTc.

Representative Poincare's Plot from matched patients in SQTc, NQTc and LQTc was visualized by geometry method in Figure 6. Illustration of Poincare plots compared between short, normal and long QTc were evident in Figure 6. They indicate that geometry in 

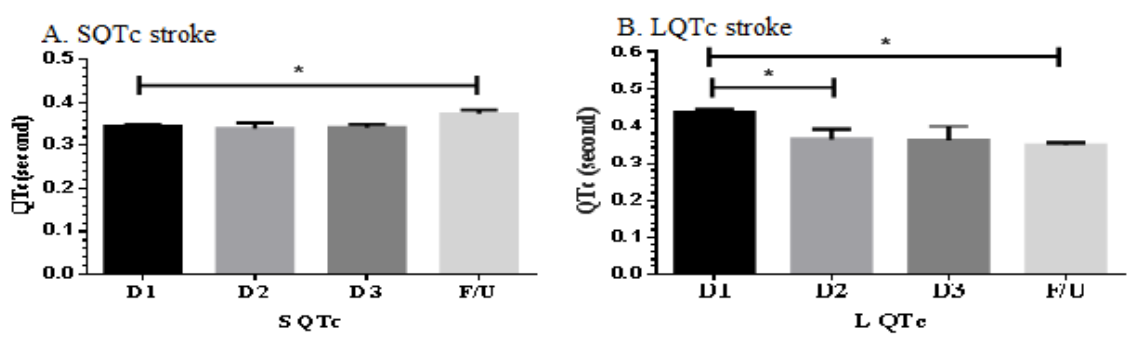

Figure 8: Illustrates QTc on D1, D2, D3 and F/U stroke in S QTc (A) and L QTc (B). " $p<0.05$

short QTc has high variation and turbulence while as in geometry of long QTc, it shows high regularity and lesser scatter gram. It is noticed that more scatter and disorganized signals are evident in $\mathrm{S}$ QTc and N QTc, respectively. For L QTc, orientation and less scatter is observed by SD1 and SD2 (11.19 vs. 48.47). These results are confirmed by sample entropy value in Figure 7.

SD1 indicates standard deviation of instantaneous R-R interval variability measured from axis 1 . SD2, standard deviation of longterm continuous R-R interval variability measured from axis 2 . The LQTc stroke patient shows a typical suppression of SD1.

The lowest value of sample entropy was obviously seen in L QTc and it showed significant difference with S QTc and N QTc (Figure 7). Less irregularity and well organized signals were proposed in L QTc. This result corresponds with visualized Poincare plot, SD2/SD1 and LF/HF ratio as well.

Since QT variation is very common characteristics after stroke, we determine QT change on day $1(\mathrm{D} 1)$, day 2 (D2), day 3 (D3) and follow up in 12 weeks later $(\mathrm{F} / \mathrm{U})$ as shown in Figure 8. Stroke recovery will occur between 3-12 weeks and it is highly dependent on the individual. S QTc was slightly increased on F/U stroke and showed significant difference among D1, D2 and D3 stroke. Interestingly, L QTc was gradually reduced from D1 to F/U stroke and showed significant difference (D1 vs. D2, D1 vs. D3 and D1 vs. F/U). These results indicate that S QTc and L QTc are not persistent after stroke. It may be from autonomic control shift and its shift does not strictly restrain.

\section{Discussion}

Since large artery ischemic stroke is closely related with vascular inflammation from oxidative stress of high risk diseases such as hypertension, atherosclerosis, atrial fibrillation, cardiac arrhythmia event might be further subsequent of these effect and also from advancing effect of an ischemia in stroke. The cortical network and insular lobe function of subcortical area injury during large artery ischemic stroke have been reported [11]. Ischemic insults produce an excess amount of free radicals, especially in the reperfused ischemic area [35]. Oxidative stress causes neuronal necrosis and death. During ischemic stroke, progressive neuron death is closely related with glia activation and neurovascular unit especially cerebral endothelium which both of them play a vital role for energy supply and reducing glutamate cascade toxicity [36]. Neurovascular-glia interaction is an important mechanism during ischemia especially under overwhelmed oxidative stress condition. Nitric oxide is well known vasomotor tone mediator from vascular endothelium and also neuromodulator of glutamate release in brain [36]. Recently, it has been reported that NO plays a vital role at medullary neuron with $\mathrm{H}_{2} \mathrm{O}_{2}$ as neuromodulator of redox signal from ROS formation and they are related with sympathetic activity drive at medulla for central hypertension [37]. Recently, $\mathrm{NO}$ and $\mathrm{H}_{2} \mathrm{O}_{2}$ are coupled relaxing factors in brain corresponding with increasing cerebral blood flow during cerebrovascular reactivity in normal and lacunar stroke (small artery stroke) [19, 32-38]. Both of them are gases that diffuse rapidly to cerebrospinal fluid and then in blood circulation within 30 seconds as assessed by cerebrovascular reactivity [38]. Moreover during global brain ischemia from cardiac arrest and treated with mild hypothermia to reducing brain mechanism, circulating $\mathrm{NO}$ and $\mathrm{H}_{2} \mathrm{O}_{2}$ play a vital role in survivor group with correspondent of improving ANS [39]. In this study, large artery ischemic stroke have been showed profound lower $\mathrm{NO}$ and an increasing $\mathrm{H}_{2} \mathrm{O}_{2}$ levels which they are similar to small artery stroke that we have reported recently. It has been recently reported that nitrite, a major metabolite of $\mathrm{NO}$ is high persistently in stroke [40]. Lower NO during stroke may be resulted from peroxynitrite formation which it is derived from $\mathrm{NO}$ and superoxide reaction. $\mathrm{H}_{2} \mathrm{O}_{2}$ is stable reactive oxygen species (ROS) and causes relaxations of large cerebral arteries [41].It has been reported that microglia proliferation following brain damage is regulated by $\mathrm{H}_{2} \mathrm{O}_{2}$ from NADPH oxidase [21]. Recently, ROS may raise blood pressure via activation of the sympathetic nervous system [22]. This finding indicates $\mathrm{H}_{2} \mathrm{O}_{2}$ influences such as increasing cerebral flow during acute stroke. As we mention above, a big source of $\mathrm{H}_{2} \mathrm{O}_{2}$ release in brain injury is from glia activation. Meanwhile, a low value of NO in the both groups indicates a limit amount of NO for glutamate release in glutamate cascade toxicity and transformation to be peroxynitrite as in ROS/RNS pathway. Moreover, $\mathrm{H}_{2} \mathrm{O}_{2}$ acts on ventricular tachyarrhythmias resulting from INa, Ito and ICa, L Channelopathy [42]. Basically, the mechanisms responsible for cardiac arrhythmias may be divided into disorders of impulse formation such as triggered activity, early afterdepolarization, delayed afterdepolarization and disorders of impulse conduction such as reentry or both disorders [43].

As shown in Table 1, there is no significant difference of carotid stenosis (NSSF vs. SSF) and the thickness of media / intima ratio among three groups of QTc. Moreover, matching control study of all parameter is clearly designed including age, sex, risk factors, hyperlipidemia, carotid stenosis and medical history. This design study opens the insight way exploring QT interval changes during stroke. On D1 stroke, a lowest level of NO in L QTc group is evident associated with a highest level of $\mathrm{H}_{2} \mathrm{O}_{2}$ compared with S QTc. This result indicates degree of oxidative stress occurring on D1 stroke 
resulting from brain injury. As many previous reports have mentioned that further brain injury causes in turn, more glia activation [36,37] Moreover, heart rate in L QTc increases and shows a significant difference compared with S QTc and N QTc. This finding indicates ventricular repolarization variation which it is under parasympathetic drive and in LQTc, suppressive activity is evident explaining from high LF and SD1. Interestingly, greatest sympathovagal balance in L QTc is clearly seen by quantitation and qualitative values of LF/HF ratio and SD2/SD1, correspondent with less irregularity signals by SampEn value and scatter gram plot of SD1 vs. SD2 in Poincare' plot. Higher levels of SBP, DBP, HR, PP, and MABP with low PTT level in L QTc indicate increasing cardio-hemodynamic responses from greater sympathetic drive and lesser parasympathetic drive which reflect the baroreceptor reflex. During acute stroke on D1, ANS modulation through sympathetic predominant with low parasympathetic drive is strongly evident by non-linear function of HRV (SD1, SD2, LF and $\mathrm{HF}$ ), not by linear function (pNN 50(\%)). An increased pNN 50(\%) of L QTc with significant difference compared with S QTc represents total vagal outflow indicating quantitation of sympathovagal balance but for QT interval variation, time domain analysis is limited. As we know that ventricular repolarization of the first cycle affects the next $\mathrm{P}$ wave in the second EKG tracing cycle, we excluded those AF in this study and then we determined changes of QTc variation on D1, D2, D3 and F/U stroke. This result indicates changes in QTc towards normal QTc resulting from compromising ANS modulation and brain-heart axis shift.

\section{Conclusion}

Increasing sympathetic activity with suppression of parasympathetic drive during acute stroke in LQTc is the important key determinant of autonomic modulation and derangement in brain-heart axis shift. Neurovascular oxidative stress through $\mathrm{NO}$ and $\mathrm{H}_{2} \mathrm{O}_{2}$ play a role in ventricular arrhythmias.

\section{Acknowledgement}

This work was supported in part by National Research University Project of Thailand, Office of Higher Education Commission and in part by faculty of medicine, Thammasat University.

\section{References}

1. Barron SA, Rogovski Z, Hemli J. Autonomic consequences of cerebral hemisphere infarction. Stroke. 1994; 25: 113-116.

2. Oppenheimer SM, Cechetto DF, Hachinski VC. Cerebrogenic cardiac arrhythmias: cerebral electrocardiographic influences and their role in sudden death. Arch Neurol. 1990; 47: 513-519.

3. E Kossi MM, Zakhary MM. Oxidative stress in the context of acute cerebrovascular stroke. Stroke. 2000; 31: 1889-1892.

4. Sörös P, Hachinski V. Cardiovascular and neurological causes of sudden death after ischaemic stroke. Lancet Neurol. 2012; 11: 179-188.

5. Constantinescu V, Matei D, Cuciureanu D, Corciova C, Ignat B, Popescu CD. Cortical modulation of cardiac autonomic activity in ischemic stroke patients. Acta Neurol Belg. 2016; 116: 473-480

6. Jørgensen HS, Nakayama H, Reith J, Raaschou HO, Olsen TS. Acute stroke with atrial fibrillation. The Copenhagen Stroke Study. Stroke. 1996; 27: 17651769.

7. Goldstein DS. The electrocardiogram in stroke: relationship to pathophysiological type and comparison with prior tracings. Stroke. 1979; 10 : 253-259.
8. Sander D, Winbeck K, Klingelhofer J, Etgen T, Conrad B. Prognostic relevance of pathological sympathetic activation after acute thromboembolic stroke. Neurology. 2001; 57: 833-838.

9. Intharakham K, Suwanprasert K, Muengtaweepongsa S. Correlation between decreased parasympathetic activity and reduced cerebrovascular reactivity in patients with lacunar infarct. Curr Neurovasc Res. 2017; 14: 65-70.

10. Taggart P. Brain-heart interactions and cardiac ventricular arrhythmias. Neth Heart J. 2013; 21: 78-81.

11. Tahsili-Fahadan P, Geocadin RG. Heart-Brain Axis: Effects of Neurologic Injury on Cardiovascular Function. Circ Res. 2017; 120: 559-572.

12. Seely AJ, Macklem PT. Complex systems and the technology of variability analysis. Crit Care. 2004; 8: R367-384

13. Rangsungnoen $S$, Chanbenjapipu $P$, Mathuradavong $N$, Suwanprasert $K$. A hybrid signal processing of RR intervals from QTc variation searching arrhythmia and improving Heart Rate Variability assessment in acute large artery ischemic stroke. Journal of Medical Engineering. 2016.

14. Sungnoon R, Suwanprasert K, Muengtaweepongsa S. Atrial electrophysiological property analysis by sample entropy and atrial fibrillatory rate with cardiac autonomic derangements in acute ischemic stroke with atrial fibrillation. Neurol Asia. 2014; 19: 11-18.

15. Villa A, Bacchetta A, Milani O, Omboni E. QT interval prolongation as predictor of early mortality in acute ischemic stroke patients. Am J Emerg Med. 2001; 19: 332-333.

16. Wang $Q$, Tang $X N$, Yenari MA. The inflammatory response in stroke. J Neuroimmunol. 2007; 184: 53-68.

17. Dormanns K, Brown RG, David T. The role of nitric oxide in neurovascular coupling. J Theor Biol. 2016; 394: 1-17.

18. Chaiyawatthanananthn $\mathrm{P}$, Suwanprasert $\mathrm{K}$, Muengtaweepongsa $\mathrm{S}$ Differentiation of serum sLOX-1 and NO levels in acute ischemic stroke patients with internal carotid artery stenosis and those without internal carotid artery stenosis. J Med Assoc Thai. 2016; 99: S48-S53.

19. Intharakham K, Suwanprasert K. Neurovascular coupling assessed by Heart Rate Variability (HRV), circulating nitric oxide and hydrogen peroxide during Cerebrovascular Reactivity. In Govitrapong P editor, IBRO-APRC Associate School of Neuroscience 2013 and Proceedings of 17th Thai Neuroscience Society International Conference. 2013; 2013: 66-72.

20. lida Y, Katusic ZS. Mechanisms of cerebral arterial relaxations to hydrogen peroxide. Stroke. 2000; 31: 2224-2230.

21. Mander PK, Jekabsone A, Brown GC. Microglia proliferation is regulated by hydrogen peroxide from NADPH oxidase. J Immunol 2006; 176: 1046-1052.

22. Campese VM, Ye S, Zhong H, Yanamadala V, Ye Z, Chiu J. Reactive oxygen species stimulate central and peripheral sympathetic nervous system activity. Am J Physiol Heart Circ Physiol. 2004; 287: H695-H703.

23. Sovari AA. Cellular and Molecular Mechanisms of Arrhythmia by Oxidative Stress. Cardiol Res Pract. 2016; 2016: 9656078

24. Boo YC, Tressel SL, Jo H. An improved method to measure nitrate/nitrite with an NO-selective electrochemical sensor. Nitric Oxide. 2007; 16: 306-312.

25. Lacy F, O'Connor DT, Schmid-Schönbein GW. Plasma hydrogen peroxide production in hypertensives and normotensive subjects at genetic risk of hypertension. J Hypertens. 1998; 16: 291-303.

26. Baldassarre D, Amato M, Bondioli A, Sirtori CR, Tremoli E. Carotid artery intima-media thickness measured by ultrasonography in normal clinical practice correlates well with atherosclerosis risk factors. Stroke. 2000; 31: 2426-2430.

27. Minoretti P, Politi P, Martinelli V, Emanuele E, Bertona M, Falcone C, et al. QT interval duration in apparently healthy men is associated with depressionrelated personality trait neuroticism. J Psychosom Res. 2006; 61: 19-23.

28. Viskin S, Postema PG, Bhuiyan ZA, Rosso R, Kalman JM, Vohra JK, et al. The response of the QT interval to the brief tachycardia provoked by standing: a bedside test for diagnosing long QT syndrome. J Am Coll Cardiol. 2010; 55: 1955-1961. 
29. Bigger JT, Fleiss JL, Rolnitzky LM, Steinman RC. The ability of severa short-term measures of RR variability to predict mortality after myocardia infarction. Circulation. 1993; 88: 927-934.

30. Tarvainen MP, Niskanen JP, Lipponen JA, Ranta-Aho PO, Karjalainen PA Kubios HRV-- heart rate variability analysis software. Comput Methods Programs Biomed. 2014; 113: 210-220.

31. Gesche H, Grosskurth D, Küchler G, Patzak A. Continuous blood pressure measurement by using the pulse transit time: comparison to a cuff-based method. Eur J Appl Physiol. 2012; 112: 309-315.

32. Kamen PW, Krum H, Tonkin AM. Poincare plot of heart rate variability allows quantitative display of parasympathetic nervous activity in humans. Clin Sci (Lond). 1996; 91: 201-208.

33. Brennan M, Palaniswami $M$, Kamen $P$. Poincaré plot interpretation using a physiological model of HRV based on a network of oscillators. Am J Physiol Heart Circ Physiol. 2002; 283: H1873-1886.

34. Brennan M, Palaniswami M, Kamen $P$. Do existing measures of Poincare plot geometry reflect nonlinear features of heart rate variability?. IEEE Trans Biomed Eng. 2001; 48: 1342-1347.

35. Hu X, De Silva TM, Chen J, Faraci FM. Cerebral Vascular Disease and Neurovascular Injury in Ischemic Stroke. Circ Res. 2017; 120: 449-471.

36. Terasaki Y, Liu Y, Hayakawa K, Pham LD, Lo EH, Ji X, et al. Mechanisms of neurovascular dysfunction in acute ischemic brain. Curr Med Chem. 2014 21: $2035-2042$
37. Chan SH1, Chan JY. Brain stem oxidative stress and its associated signaling in the regulation of sympathetic vasomotor tone. J Appl Physiol (1985). 2012; 113: $1921-1928$

38. Intharakham K, Suwanprasert K. Complexity of autonomic control during cerebrovascular reactivity. Biomedical Engineering International Conference (BMEiCON-2013). 2013; 23-25

39. Jantanukul A, Muengtaweepongsa S, Suwanprasert K. Suppression of autonomic drive determined by nonlinear HRV analysis in therapeutic hypothermia after cardiac arrest. Biomedical Engineering International Conference (BMEiCON-2013). 2013; 23-25.

40. Rashid PA, Whitehurst A, Lawson N, Bath PM. Plasma nitric oxide (nitrate/ nitrite) levels in acute stroke and their relationship with severity and outcome. JStroke Cerebrovasc Dis. 2003; 12: 82-87.

41. lida Y, Katusic ZS. Mechanisms of cerebral arterial relaxations to hydrogen peroxide. Stroke. 2000; 31: 2224-2230.

42. Wang L, Sun L, Zhang Y, Wu H, Li C, Pan1 Z, et al. Ionic mechanisms underlying action potential prolongation by focal cerebral ischemia in rat ventricular myocytes. Cell Physiol Biochem. 2009; 23: 305-316.

43. Gaztañaga L, Marchlinski FE, Betensky BP. Mechanisms of cardiac arrhythmias. Rev Esp Cardiol (Engl Ed). 2012; 65: 174-185.
Austin J Cerebrovasc Dis \& Stroke - Volume 4 Issue 5 - 2017 ISSN : 2381-9103 | www.austinpublishing group.com

Suwanprasert et al. () All rights are reserved
Citation: Suwanprasert K, Chanbenjapipu P and Muengtaweepongsa S. Neurovascular Oxidative Stress and Autonomic Modulation Contributing to OT Interval Variations in Acute Large Artery Ischemic Stroke. Austin J Cerebrovasc Dis \& Stroke. 2017; 4(5): 1071. 